\title{
Influence des paramètres de composition sur le comportement du béton autoplaçant à l'état frais
}

\author{
N. BOUHAMOU ${ }^{*}$, N. BELAS ${ }^{1}$, H. MESBAH ${ }^{2}$, A. MEBROUKI ${ }^{1}$ et A. YAHIA ${ }^{3}$ \\ ' Faculté des sciences de l'ingénieur, Université Abdelhamid Ibn Badis, Mostaganem, \\ (27000), Algérie \\ ${ }^{2}$ Institut National des Sciences Appliquées de Rennes, 20 avenue des Buttes de Coësmes \\ CS 14315-35043 Rennes Cedex, France. \\ ${ }^{3}$ Université de Sherbrooke, Québec, Canada
}

*Correspondance,courriel:nbouhamov@yahoo.fr

\section{Résumé}

La présente étude s'inscrit dans le cadre des travaux de recherche menés au département de génie civil de l'université de Mostaganem, en Algérie. Elle concerne l'étude de l'influence des paramètres de composition sur le comportement du béton autoplaçant à l'état frais. Elle a pour objectif de comprendre les rôles joués par les différents constituants susceptibles d'entrer dans la formulation d'un BAP à base de matériaux locaux notamment le sable de mer et le sable de carrière (considéré comme déchet naturel) et de mettre en évidence les éventuelles interactions entre constituants. Les résultats obtenus offrent de belles perspectives pour optimiser les BAP. Nos essais ont permis de développer une variété de formulations de béton autoplaçant répondant aux critères rhéologiques (bonne déformabilité, moins de ressuage, absence de ségrégation).

Mots-clés : Béton autoplaçant, superplastifiant, fines calcaires, matériaux locaux, béton frais.

\section{Abstract \\ Effect of Composition Parameters on Fresh State Properties of Self- Compacting Concrete}

This present study is enrolled according to the plan of research activities led at civil engineering department of Mostaganem University. It concerns the study of the effect of 
some arrangement parameters upon the self-compacting concrete at it's fresh state. The purpose of this research is to understand the roles of the different components susceptible to take part in formulating a SCC based on local materials, in particular sand of beach and sand of quarry (which is considered as natural loss); and to set clearly the eventual interaction between the components. The results that have been obtained offer very well perspectives to optimize the $\mathrm{SCC}$. Our tests, also have allowed improving a variety of formulas of $S C C$ responding to rheologic criteria.

Keywords : Self-compacting concrete, superplasticizer, limestone powder, local materials, fresh concrete.

\section{Introduction}

Dans les années quatre-vingt et quatre-vingt-dix, les études relatives aux bétons à hautes performances ont montré le rôle néfaste de l'excès d'eau dans les bétons. La réduction de cette quantité d'eau, par emploi de défloculants et par correction de l'empilement granulaire via les ultrafines, a conduit aux gains de résistance et de durabilité que l'on connaît [1]. En prolongement de ces travaux scientifiques, l'amélioration constatée de l'ouvrabilité de ces nouveaux bétons a conduit les chercheurs à développer et à fiabiliser cette propriété.

Aujourd'hui, en totale continuité avec les bétons à hautes performances, c'est un changement d'objectifs constituant une véritable révolution culturelle que proposent les bétons autoplaçants : l'étude du matériau n'est plus seulement gouvernée par l'amélioration de la résistance et de la pérennité. Toutefois ces dernières propriétés restent calées à des niveaux équivalents ou supérieurs à celles des bétons courants. Ce sont désormais, avec les bétons autoplaçants, les aptitudes à être aisément mis en œuvre sans vibration qui sont devenues prioritaires. Ces aptitudes vont avoir de grandes conséquences en termes de délai d'exécution, de réduction de matériels, de qualité de bétonnage, de facilité de mise en œuvre, de respect du voisinage et de moindre pénibilité pour les ouvriers [2].

Le présent travail a pour objectifs de comprendre l'influence des principaux constituants sur le comportement des bétons autoplaçants à l'état frais, et avant tout à chercher des solutions pour optimiser une formule de béton autoplaçant à base de matériaux locaux et non d'expliquer les différences entre béton autoplaçant (BAP) et béton ordinaire (BO). 


\section{Matériel et méthodes}

\section{2-1. Matériaux utilisés}

Leurs caractéristiques sont données par le Tableau 1.

Tableau 1 : Caractéristiques des matériaux utilisés

\begin{tabular}{|c|c|c|c|c|c|c|c|c|c|}
\hline \multirow[b]{2}{*}{ Matériau } & \multirow{2}{*}{$\begin{array}{l}\text { Sable } \\
\text { de mer } \\
\text { (Sm) }\end{array}$} & \multirow{2}{*}{$\begin{array}{l}\text { Sable } \\
\text { de } \\
\text { carrière } \\
\text { (Sc) } \\
\end{array}$} & \multirow{2}{*}{$\begin{array}{l}\text { Gravier } \\
3 / 8 \\
(G 3 / 8)\end{array}$} & \multirow{2}{*}{$\begin{array}{l}\text { Gravier } \\
8 / 15 \\
(G 8 / 15)\end{array}$} & \multirow{2}{*}{$\begin{array}{l}\text { Gravier } \\
15 / 20 \\
(G 15 / 20)\end{array}$} & \multirow[b]{2}{*}{$\begin{array}{l}\text { Fines } \\
\text { (F) }\end{array}$} & \multirow{2}{*}{$\begin{array}{l}\text { Ciment } \\
\text { (C) }\end{array}$} & \multicolumn{2}{|l|}{ Adjuvant } \\
\hline & & & & & & & & BV40 & $\begin{array}{l}\text { 20HE } \\
\text { (Sp) }\end{array}$ \\
\hline Nature & $\begin{array}{l}\text { Siliceux } \\
0 / D= \\
0 / 2\end{array}$ & $\begin{array}{l}\text { Calcaire } \\
0 / D= \\
0 / 4\end{array}$ & Calcaire & Calcaire & Calcaire & Calcaires & $\begin{array}{l}\text { CPJ } \\
\text { CEMII } \\
\int_{32,5} A\end{array}$ & Plastifiant & $\begin{array}{l}\text { Viscosant } \\
\text { et } \\
\text { fluidifiant }\end{array}$ \\
\hline
\end{tabular}

\section{2-2. Méthode de formulation}

Cinq bétons autoplaçants et un béton vibré (ordinaire) ont été mis en œuvre. Le critère de comparaison choisi étant la résistance mécanique en compression à 28 jours. La méthode de formulation utilisée pour concevoir les compositions de BAP testées dans cette étude expérimentale est une méthode empirique basée sur quatre points :

- La formulation du BAP doit répondre aux critères de résistance mécanique (fixés ou désirés) choisis à partir de la formule de Bolomey [3].

- Le volume de pâte doit favoriser l'écoulement du béton tout en réduisant le coût en matières premières de cette formulation [4].

Pour cela, le volume de pâte (eau efficace+air occlus +ciment+additions +adjuvant+ fines des granulats de dimension inférieure à $80 \mu \mathrm{m}$ ) est fixé à 375 litres. L'eau efficace correspond à l'eau totale théorique (eau de gâchage+eau apportée par les granulats+ eau apportée par les adjuvants) à laquelle on soustrait l'eau absorbée par les granulats. - Le dosage en adjuvant (superplastifiant et viscosifiant) est calculé afin de limiter la ségrégation et le ressuage [5]. Ce dosage est déterminé de manière expérimentale à partir d'essais sur bétons frais pour lequel l'étalement doit être compris entre 60 et $75 \mathrm{~cm}$.

- L'optimisation de la constitution doit réduire la ségrégation et favoriser l'écoulement. Pour cela et afin de sélectionner une combinaison adéquate de granulats qui améliore la maniabilité et la déformabilité du béton autoplaçant, plusieurs proportions ont été considérées tout en gardant un rapport gravier sur sable (G/S) proche de 1. 
- En ce qui concerne la formulation du béton vibré correspondant, sa composition a été obtenue à partir de la méthode de Dreux-Gorisse en conservant le même rapport eau sur ciment $(\mathrm{E} / \mathrm{C})$ que celui des BAP.

Les bétons ont été confectionnés selon les recommandations actuelles de l'Association Française de Génie Civil (AFGC) [6].

Toutes les compositions étudiées sont présentées dans le Tableau 2.

Tableau 2 : Composition des différents bétons

\begin{tabular}{|l|c|c|c|c|c|c|}
\hline \multirow{2}{*}{$\begin{array}{c}\text { Compositions } \\
\left(\mathrm{kg} / \mathrm{m}^{3}\right)\end{array}$} & \multicolumn{7}{c|}{ Désignation des bétons } \\
\cline { 2 - 7 } & BAP l & BAP 2 & BAP 3 & BAP 4 & BAP 5 & B0 \\
\hline G/S & 0,8 & 0,9 & 1,0 & 1,1 & 1,2 & 1,6 \\
\hline Ciment $\left(\mathrm{Kg} / \mathrm{m}^{3}\right)$ & 400 & 400 & 400 & 400 & 400 & 350 \\
\hline Fillers calcaires $\left(\mathrm{Kg} / \mathrm{m}^{3}\right)$ & 100 & 100 & 100 & 100 & 100 & - \\
\hline Sable de mer $\left(\mathrm{Kg} / \mathrm{m}^{3}\right)$ & 578 & 578 & 578 & 495 & 495 & 129 \\
\hline Sable de carrière $\left(\mathrm{Kg} / \mathrm{m}^{3}\right)$ & 333 & 299 & 249 & 299 & 249 & 576 \\
\hline Gravillon 3/8 $\left(\mathrm{Kg} / \mathrm{m}^{3}\right)$ & 333 & 366 & 333 & 333 & 416 & 204 \\
\hline Gravillon 8/15 $\left(\mathrm{Kg} / \mathrm{m}^{3}\right)$ & 416 & 416 & 499 & 532 & 499 & 947 \\
\hline Plastiment BV $40\left(\mathrm{Kg} / \mathrm{m}^{3}\right)$ & - & - & - & - & - & 5,25 \\
\hline Viscocrète $20 \mathrm{HE}\left(\mathrm{Kg} / \mathrm{m}^{3}\right)$ & 4 & 4 & 4 & 4 & 4 & - \\
\hline Eau efficace $\left(\mathrm{Kg} / \mathrm{m}^{3}\right)$ & 200 & 200 & 200 & 200 & 200 & 175 \\
\hline E/C & 0,5 & 0,5 & 0,5 & 0,5 & 0,5 & 0,5 \\
\hline
\end{tabular}

\section{2-3. Comparaison des formulations étudiées}

\section{2-3-1. Caractérisation des bétons}

La caractérisation à l'état frais des bétons s'est limitée aux essais recommandés par l'AFGC [6], à savoir l'étalement au cône, l'écoulement à la boîte en $\|\boldsymbol{L}\|$ et la stabilité au tamis et ressuage.

\section{2-3-I-I. L'essai d'étalement (BAP) ou d'affaissement(BO)}

II s'agit de démouler un cône normalisé DIN (ou cône d'Abrams) de béton frais et de mesurer le diamètre de la galette de béton obtenue (Photo I). II faut rappeler qu'est considéré comme autoplaçant, un béton qui forme une galette d'un diamètre compris entre 60 et 75 centimètres. Pour les BAP, des temps d'écoulement intermédiaires ont été mesurés. II s'agit du temps nécessaire pour atteindre un diamètre de $50 \mathrm{~cm}$, noté $\mathrm{t}_{50}[6]$. 


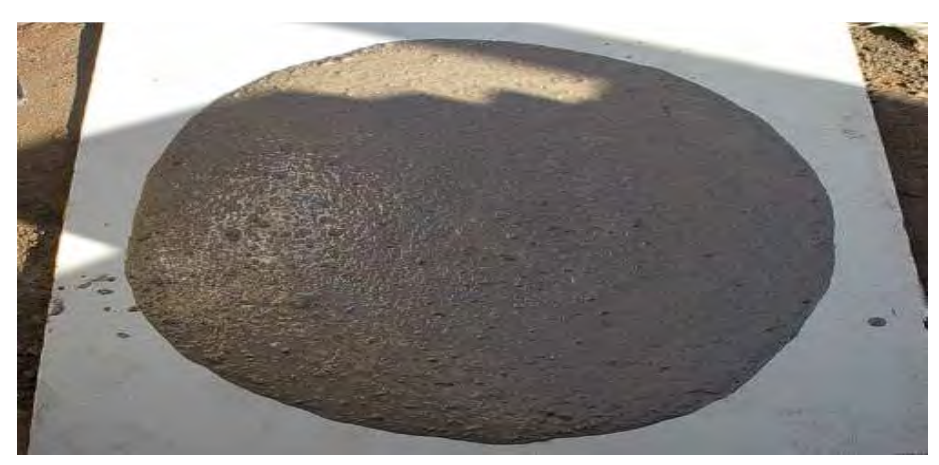

Photo 1 : Essai d'étalement

Pour la formulation vibrée (BO), c'est le test traditionnel du cône d'Abrams selon la norme NF P 18-451 qui s'est substitué à celui d'étalement.

\section{2-3-1-2. L'essai de la boite en II L II}

La boite en II $L$ // permet de tester la mobilité du béton en milieu confiné et de vérifier que sa mise en place ne sera pas contrariée par des phénomènes de blocage.

13 litres de béton sont mis dans la partie verticale puis on laisse le béton se reposer pendant 1 minute. La trappe est ensuite levée et le béton s'écoule dans la partie horizontale de la boite à travers le ferraillage. A la fin de l'écoulement, on mesure les hauteurs $H_{1}, H_{2}$ et le résultat est exprimé en termes de taux de remplissage $H_{2} / H_{1}$ (Figure 1, Photo 2)[6].

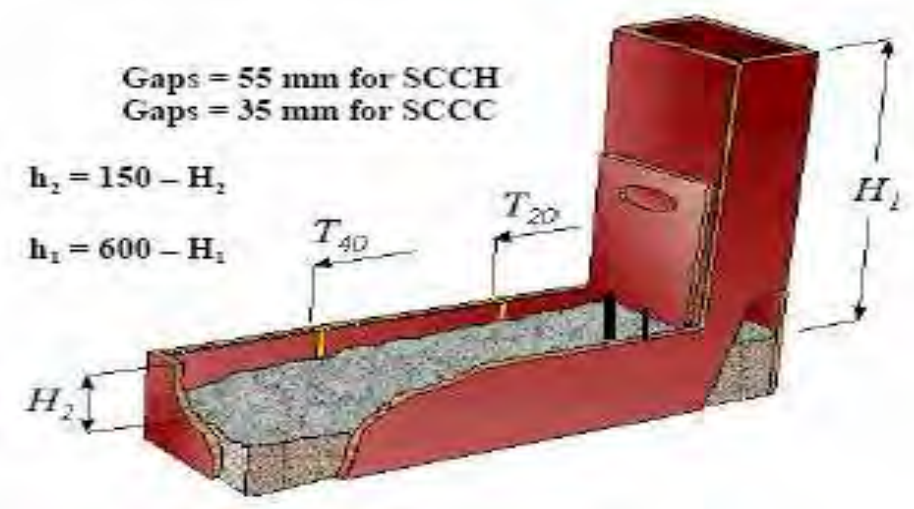

Figure 1 : Mesure des paramètres après stabilisation 


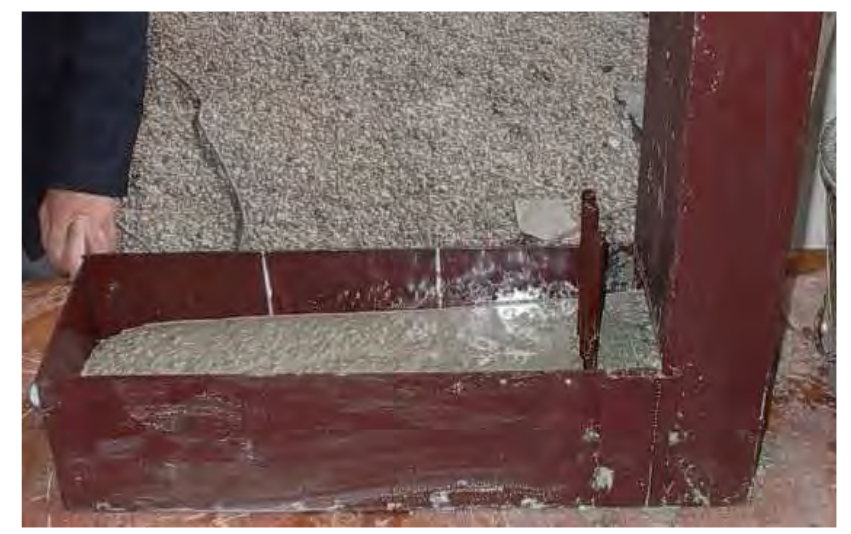

Photo 2 : Remplissage du béton à l'amont des armatures

\section{2-3-1-3. L'essai de stabilité au tamis}

II consiste à mesurer la proportion $\boldsymbol{\pi}$ d'éléments fins de béton (laitance) passant au travers d'un tamis d'ouverture $5 \mathrm{~mm}$ (Photo 3) pour qualifier le béton vis-à-vis du risque de ségrégation et de déduire si le béton testé possède une stabilité satisfaisante ou non. Les critères d'acceptabilité d'une formulation de BAP sont divisés en trois classes [6]:

- $0 \%<\boldsymbol{\pi}$ (laitance) $<15 \%$ : stabilité satisfaisante,

- $15 \%<\pi$ (laitance) $<30 \%$ : stabilité critique

- $\boldsymbol{\pi}$ (laitance) $>30 \%$ : stabilité très mauvaise (ségrégation systématique, béton inutilisable).

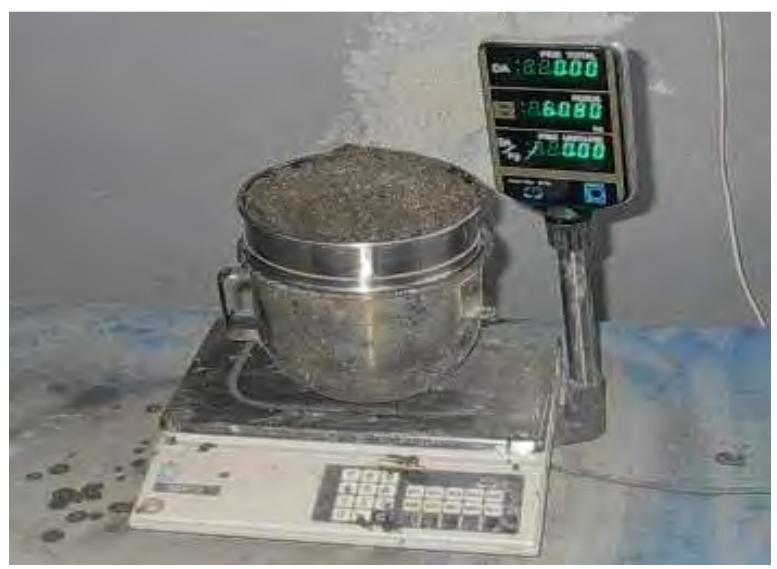

Photo 3 : Essai de stabilité au tamis

N. BOUHAMOU et al. 


\section{2-3-1-4. L'essai de ressuage}

Pour l'essai de ressuage, il s'agit de mesurer la quantité de liquide remonté à la surface d'une éprouvette $15 \times 30 \mathrm{~cm}$ au bout de $3 \mathrm{~h}$ (Figure 2).

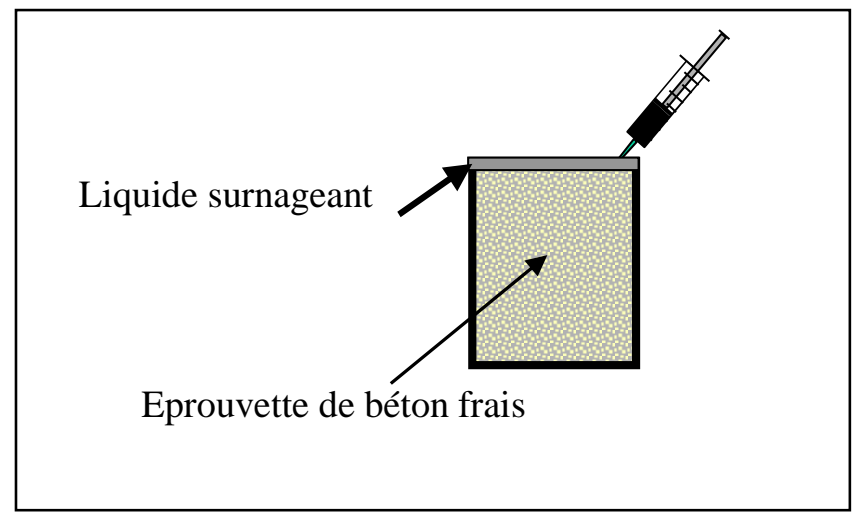

Figure 2 : Essai de ressuage

\section{2-3-1-5. L'air occlus}

Avec un appareillage spécifique, l'aéromètre, la teneur en air des bétons est déterminée selon la norme NF P 18-353. Le principe est de remplir un volume déterminé de béton, de mettre en pression le récipient hermétiquement clos situé au dessus, mettre en contact les deux récipients et on accède directement à la valeur de la teneur en air occlus. Les échantillons utilisés pour déterminer la résistance mécanique en compression des différents bétons étudiés, sont des éprouvettes cylindriques de diamètre $11 \mathrm{~cm}$ et de hauteur $22 \mathrm{~cm}$. Une fois démoulées, elles subissent une rectification et sont conservées dans l'eau dans une salle à $20^{\circ} \mathrm{C}$ et $95 \pm 5 \%$ d'humidité relative jusqu'à 28 jours.

\section{2-3-2. Résultats et analyse}

Les réponses des tests de caractérisation réalisés sur les bétons confectionnés sont présentées dans le Tableau 3.

Nous pouvons constater que l'ensemble des bétons autoplaçants respecte le critère d'étalement. L'étalement spécifié est respectivement compris entre $70 \mathrm{~cm}$ (BAP3) et 74,5 cm (BAP5).

Bien qu'aucune limite ne soit donnée pour les temps d'étalement, les temps mesurés pour atteindre une galette de $50 \mathrm{~cm}$ de diamètre $\left(t_{50}\right)$ sont proches des valeurs couramment rencontrées (3 secondes) [6]. 
Tableau 3 : Caractérisation des bétons d'étude (BAP et BO)

\begin{tabular}{|c|c|c|c|c|c|c|c|}
\hline ESSAIS & BETONS & BAP 1 & BAP 2 & BAP 3 & BAP 4 & BAP 5 & $\mathrm{BO}$ \\
\hline \multicolumn{2}{|c|}{ AFFAISSEMENT (cm) } & - & - & - & - & - & 8 \\
\hline \multirow{2}{*}{ ETALEMENT } & $\operatorname{Dmoy}(\mathrm{cm})$ & 73,0 & 72,0 & 70,0 & 71,0 & 74,5 & - \\
\hline & $t_{50}(s)$ & 2,5 & 2,3 & 2,0 & 2,0 & 2,8 & - \\
\hline \multirow{2}{*}{ BOITE EN L } & $\mathrm{H} 2 / \mathrm{HI}$ & 0,93 & 0,91 & 0,87 & 0,89 & 0,95 & - \\
\hline & $\mathrm{T}_{40}(\mathrm{~s})$ & 3,0 & 3,1 & 3,5 & 3,3 & 3,0 & - \\
\hline \multicolumn{2}{|c|}{ STABILITE AU TAMIS $\pi(\%)$} & 11,85 & 12,60 & 7,68 & 8,45 & 13,50 & - \\
\hline \multicolumn{2}{|c|}{ RESSUAGE $(\% 0)$} & 1,46 & 1,52 & 1,15 & 1,26 & 1,58 & - \\
\hline \multicolumn{2}{|c|}{ AIR OCCLUS (\%) } & 2,8 & 2,4 & 1,9 & 2,2 & 3,2 & 1,6 \\
\hline \multicolumn{2}{|c|}{ Rc à 28 jours (MPa) } & 37,9 & 38,6 & 41,0 & 39,8 & 37,1 & 36,5 \\
\hline
\end{tabular}

Pour toutes les compositions de BAP, I'auréole de laitance à la périphérie des galettes de béton était absente ou très faible (de 1 à $2 \mathrm{~mm}$ ). De plus, les gros granulats ont toujours été entraînés correctement par la matrice cimentaire et ne sont pas restés amoncelés au milieu des galettes de béton.

Sur la consistance du béton ordinaire (vibré) : aucune condition n'avait été fixée au préalable. La classe d'affaissement obtenue est la classe $S 2$ (béton plastique : de 5 à 9 cm d'après la norme NF EN 206-1).

Sur la ségrégation dynamique des BAP, les résultats représentés par le Tableau 2 sont conformes à ce que l'on peut attendre d'un béton autoplaçant. Cependant, le plus important dans cet essai est que le béton testé s'écoule à travers les armatures correctement. Sur ce point, aucun problème n'est à signaler puisque tout les BAP présentent des taux de remplissage supérieur à 0,80 . Les valeurs du paramètre d'appréciation de l'ouvrabilité du BAP $\mathrm{T}_{40}$ sont proches de celles couramment trouvées (3 à 5 secondes).

Sur la ségrégation statique, tous les BAP ont un taux de ségrégation inférieur à $15 \%$, synonyme d'une stabilité correcte. Un ressuage important est le signe d'une dégradation de la qualité esthétique des parements et de la durabilité. La valeur limite de ressuage conseillée doit être inférieure ou égale à $3 \%$ en volume. Tous nos bétons respectent cette condition.

On peut noter que les résistances entre $B A P$ et $B O$ ne sont pas très différentes. Les 
raisons des petits écarts observés sont diverses:

- les résistances élevées des BAP sont liées au volume de pâte important.

- I'utilisation du superplastifiant "viscocrete 20HE" dans les formulations autoplaçantes peut avoir une influence bénéfique sur la résistance mécanique.

- la présence d'une quantité importante de filler calcaire comme addition dans les BAP peut également avoir une action positive sur leurs compacités et par conséquent sur leurs résistances mécaniques.

\section{2-3-3. Bilan}

Une première remarque pourrait être faite sur les performances obtenues par les bétons autoplaçants. Il est visible que l'on obtient de bons bétons en terme de résistances mécaniques (dues essentiellement à une forte compacité - adjuvantation, dosage en fines élevé). Les possibilités de variation des paramètres d'étude pour les BAP sont nombreuses (utilisation de différents dosages en additions minérales, d'agent de viscosité, de superplastifiant, variation du volume de pâte, du rapport $\mathrm{G} / \mathrm{S}$, du rapport $E / C$, etc.). Tout ceci engendre une vision au cas par cas de chaque propriété étudiée et ne permet pas de généraliser les observations effectuées à une gamme de résistance mécanique étendue. Cela étant, la meilleure façon d'assurer et d'atteindre au mieux les propriétés visées d'un BAP à l'état frais est sans doute d'attacher une importance toute particulière à l'influence des paramètres de composition et aboutir à une formulation optimale.

\section{Influence des paramètres de composition sur le comportement du BAP à l'état frais}

Nous proposons d'étudier les effets des paramètres de composition d'une formule de BAP sur les caractéristiques à l'état frais. Nous avons choisi de travailler sur le BAP3 qui a présenté les meilleurs résultats en comparaison avec les autres formules de BAP.

Les paramètres de formulation étudiés sont les suivants:

- le dosage en superplastifiant (\%): Sp,

- le rapport de la masse d'eau sur la masse de ciment : $E / C$,

- le rapport de la masse d'addition sur la masse de ciment (fines sur ciment) : $F / C$,

- la proportion de pâte [C (ciment)+F (addition)+E (eau)+Sp (superplastifiant)] du béton (\%) : $V_{\text {pâté }}$

- diamètre maximal des gravillons $(\mathrm{mm})$ : $\Phi_{\max }$. 
Les plages de chaque paramètre sont données dans le Tableau 4.

Tableau 4 : Plages des paramètres de composition d'une formule de BAP

\begin{tabular}{|l|c|c|c|}
\hline \multicolumn{1}{|c|}{ Paramètres } & Minimum & Intermédiaire & Maximum \\
\hline $\mathrm{Sp}(\%)$ & 0,5 & 1,0 & 1,5 \\
\hline $\mathrm{E} / \mathrm{C}$ & 0,4 & 0,5 & 0,6 \\
\hline $\mathrm{F} / \mathrm{C}$ & 0,20 & 0,25 & 0,30 \\
\hline $\mathrm{V}_{\text {pâte }}(\%)$ & 35 & 37,5 & 40 \\
\hline$\Phi_{\text {max }}(\mathrm{mm})$ & 15 & - & 20 \\
\hline
\end{tabular}

\section{3-1. Influence du superplastifiant}

L'optimisation des propriétés du superplastifiant utilisé, par rapport aux besoins visés, peut s'avérer un moyen très pratique pour la formulation des bétons et des pâtes de ciment.

Pour étudier l'effet de la variation du dosage en superplastifiant sur le comportement du $B A P$, le béton autoplaçant dont les proportions sont : $V p a ̂ t e=375 \mathrm{~L} / \mathrm{m}^{3}, \mathrm{E} / \mathrm{C}=0,5$ et $\mathrm{E} / \mathrm{F}$ $=0,25$ a été testé avec $0.5,1$ et $1.5 \%$ de superplastifiant (dosage par rapport à la masse de ciment).

Les résultats d'essai de caractérisation du BAP à l'état frais sont reportés sur le Tableau 5.

Tableau 5 : Influence du dosage en superplastifiant sur le comportement du BAP $\dot{a}$ l'état frais

\begin{tabular}{|l|c|c|c|}
\cline { 2 - 4 } \multicolumn{1}{c|}{} & $S p=0,5 \%$ & $S p=1 \%$ & $S p=1,5 \%$ \\
\hline Etalement $(\mathrm{cm})$ & 57,0 & 70,0 & 79,0 \\
\hline L-Box $\mathrm{H}_{2} / \mathrm{H}_{1}$ & 0,69 & 0,87 & 0,98 \\
\hline Stabilité au tamis $\pi(\%)$ & 8,02 & 7,68 & 14,56 \\
\hline Ressuage $(\% 0)$ & 0,91 & 1,15 & 2,23 \\
\hline
\end{tabular}

Le premier effet du superplastifiant est évidemment d'augmenter l'étalement du béton (Proust, 2002). Cette tendance est clairement constatée sur le Tableav 4. Un BAP avec un dosage en superplastifiant de $1,5 \%$ du poids de ciment présente une amélioration du diamètre d'étalement de $38,5 \%$ et $12,85 \%$ par rapport à ceux avec un dosage de 0,5 et $1 \%$ du poids de ciment respectivement. 
Sur la ségrégation statique : on peut noter que l'augmentation du dosage en superplastifiant a engendré une augmentation du taux de ségrégation. Ceci confirme I'influence positive de ce produit vis-à-vis de la ségrégation statique du BAP.

Sur la ségrégation dynamique : seule le BAP avec un dosage en superplastifiant de $0,5 \%$ possède un rapport $\mathrm{H}_{2} / \mathrm{H}_{1}$ inférieur à 0,8 . A cet effet on constate que pour une teneur en superplastifiant de l'ordre de $0,5 \%$ avec un rapport $\mathrm{E} / \mathrm{C}=0,5$, la confection d'un BAP est quasiment impossible.

Le volume d'eau de ressuage augmente lorsque le dosage en superplastifiant augmente. L'une des explications avancées est l'augmentation de la viscosité de l'eau due au superplastifiant (en général, un fluidifiant est plus visqueux que l'eau pure) [8]. II est connu également que le ressuage diminue lorsque la surface spécifique des fines augmente [9], ce qui est le cas en présence d'un défloculant comme le superplastifaint. Quoi qu'il en soit, les bétons les plus adjuvantés bénéficient moins de la protection contre le séchage procurée par le ressuage.

\section{3-2. Influence du rapport $E / C$}

A part son rôle majeur dans le phénomène de l'hydratation, l'eau est l'un des facteurs les plus importants au niveau de l'ouvrabilité du béton. L'augmentation du dosage en eau augmente la fluidité du béton et entraîne la diminution de la concentration en solides. Au niveau rhéologique, le seuil de cisaillement et la viscosité diminuent [10].

Cependant, l'introduction excessive d'eau provoque la chute de la résistance mécanique du béton à l'état durci, ainsi que l'apparition des phénomènes de ségrégation à l'état frais. Le dosage du ciment dans le béton est très souvent relié à ses propriétés mécaniques et sa durabilité. On considère que l'écoulement et l'ouvrabilité du béton sont reliés au rapport $\mathrm{E} / \mathrm{C}$ qui représente un facteur très influent sur ses différents aspects. Aussi la formulation des bétons ordinaires passait par l'optimisation de ce paramètre.

Pour étudier l'influence du rapport $\mathrm{E} / \mathrm{C}$ sur le comportement du BAP à l'état frais, nous avons gardé le couple volume de pâte et $\mathrm{F} / \mathrm{C}$ constants $\left(V_{\text {pâte }}=37,5 \%\right.$, Fines $/$ Ciment $=$ $0,25)$.

Les Figures 3 et 4 représentent respectivement l'influence du rapport E/C sur le diamètre d'étalement et la ségrégation dynamique.

D'après la Figure 3 , on constate que la diminution du rapport $\mathrm{E} / \mathrm{C}$ a influé directement sur l'étalement du BAP. On observe pour un BAP de rapport $\mathrm{E} / \mathrm{C}=0,4$, une diminution de diamètre d'étalement de $13 \%$ et $16 \%$ par rapport à ceux des $B A P$ de $E / C=0,5$ et 0,6 respectivement. Cette diminution est interprétée par la réduction de la quantité d'eau de gâchage incorporée lors du malaxage. 


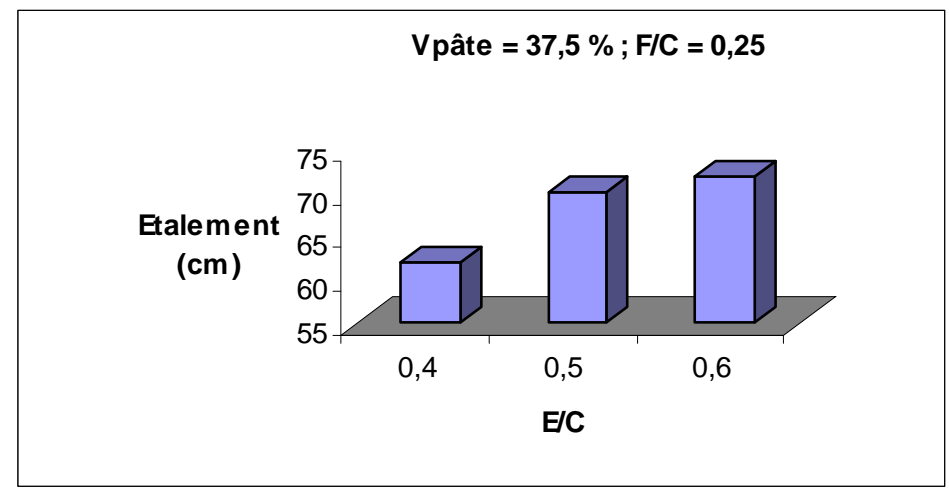

Figure 3 : Influence de la variation du rapport E/C sur le diamètre d'étalement du BAP

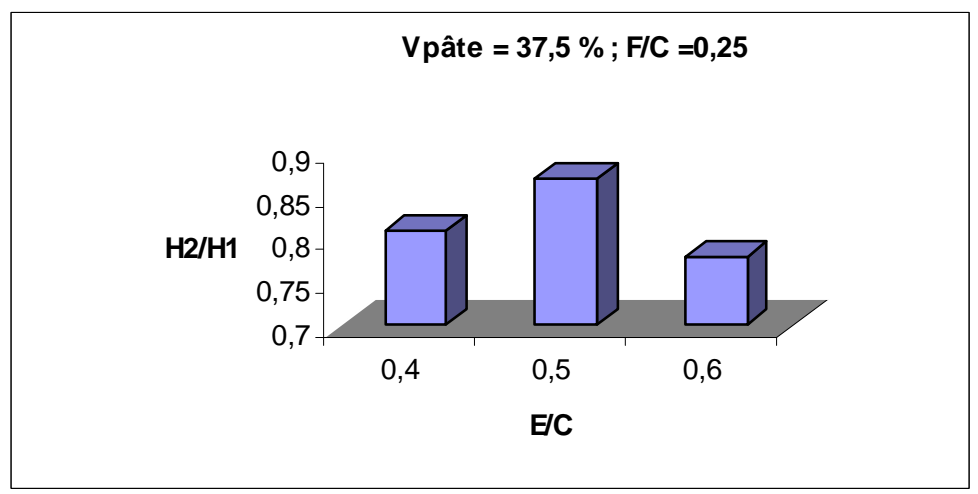

Figure 4 : Influence de la variation du rapport $E / C$ sur la ségrégation dynamique $\left(H_{2} / H_{1}\right)$

Cependant, cette diminution a influé positivement sur la stabilité et la résistance à la ségrégation dynamique pour les $\mathrm{BAP}$ de rapport $\mathrm{E} / \mathrm{C}=0,4$ et $\mathrm{E} / \mathrm{C}=0,5$ (Figure 4). Seule la formulation avec un rapport $\mathrm{E} / \mathrm{C}=0,6$ ne respecte pas cette condition ceci est dû probablement à la quantité d'eau importante.

Le Tableau 6 présente les résultats de la résistance à la ségrégation statique et du ressuage du BAP.

Tableau 6 : Influence du rapport E/C sur la résistance à la ségrégation statique et le ressuage.

\begin{tabular}{|l|c|c|c|}
\cline { 2 - 4 } \multicolumn{1}{l|}{ Caractéristique } & $\mathrm{E} / \mathrm{C}=0,4$ & $\mathrm{E} / \mathrm{C}=0,5$ & $\mathrm{E} / \mathrm{C}=0,6$ \\
\hline Stabilité au tamis $\pi(\%)$ & 7,45 & 7,68 & 15,98 \\
\hline Ressuage $(\% 0)$ & 1,09 & 1,15 & 3,25 \\
\hline
\end{tabular}


Les BAP correspondant aux rapports $\mathrm{E} / \mathrm{C}=0,4$ et 0,5 ont un taux de ségrégation inférieur à $15 \%$ et un ressuage n'excédant pas les $3 \%$, synonyme d'une stabilité correcte.

Le BAP de $E / C=0,6$ présente la particularité d'engendrer une mauvaise stabilité vis-àvis de l'écoulement.

\section{3-3. Influence du rapport $\mathrm{F} / \mathrm{C}$}

Les BAP sont caractérisés par une fluidité importante et surtout une diminution de la ségrégation et du ressuage par rapport au béton vibré. Pour obtenir ces propriétés et pour un meilleur arrangement granulaire, on ajoute de fortes teneurs en additions minérales.

Les réponses des tests (étalement, boite en $\mathrm{L}$ ) réalisés sur les bétons confectionnés sont présentées par les Figures 5 et 6.

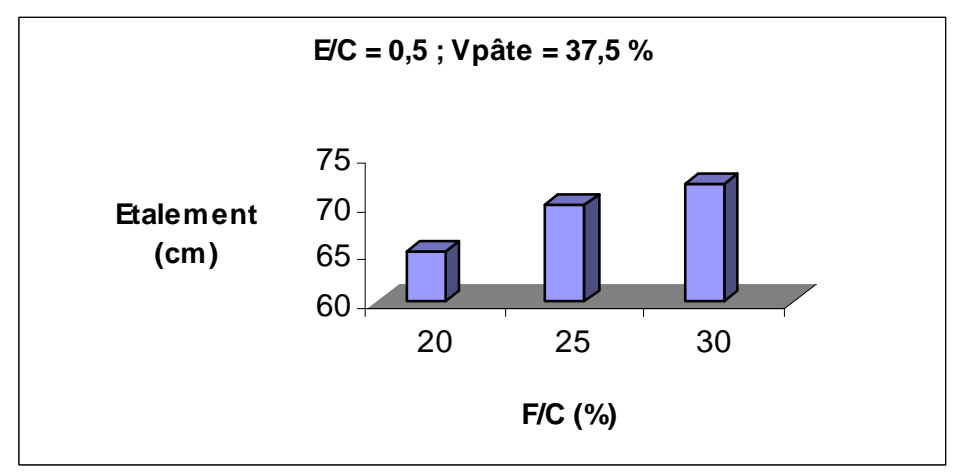

Figure 5 : Influence de la variation du rapport $F / C$ sur le diamètre d'étalement

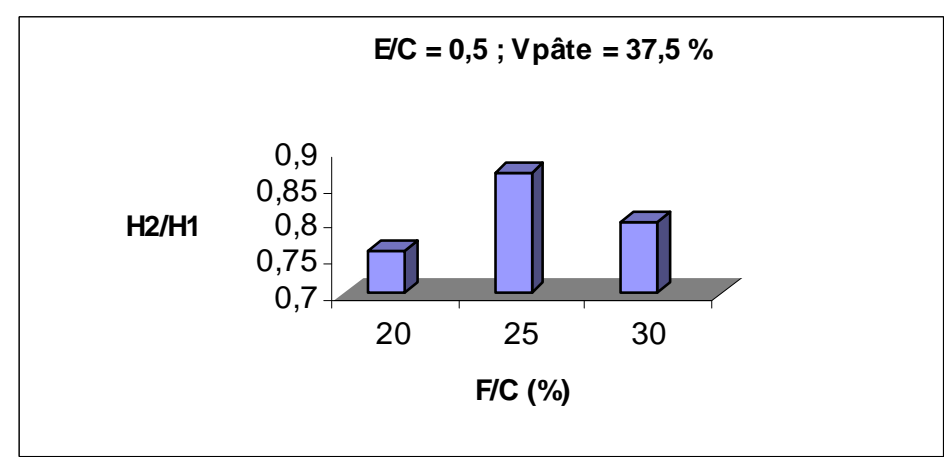

Figure 6 : Influence de la variation du rapport $F / C$ sur la ségrégation dynamique (H2/HI). 
Un seul béton présente un taux de remplissage inférieur à 0,8 (béton à $F / C=20 \%$ ), mais son étalement au cône d'Abrams est de $65 \mathrm{~cm}$; il peut être considéré comme satisfaisant et à la limite du domaine autoplaçant. Les autres bétons (à $F / C=25 \%$ et $\mathrm{F} / \mathrm{C}=30 \%$ ) ne présentent aucun signe de blocage et un écoulement continu à la boite en "L II. Leurs diamètres d'étalement et leurs taux de remplissage, laissent présumer des bétons de propriétés autoplaçantes.

Nous remarquons que le filler calcaire semble avoir une faible influence sur la demande en eau, et peut conduire à une légère diminution de viscosité du mélange cimentaire. Ceci peut justifier l'utilisation de cette addition à dosages élevés dans la formulation des $B A P$. Nous avons constaté également que pour un rapport $E / C$ et un volume de pâte constants, l'ajout du filler calcaire contribue à diminuer la viscosité du BAP et augmenter son diamètre d'étalement, avant de provoquer une augmentation de viscosité qui se traduit par une diminution du rapport $\mathrm{H}_{2} / \mathrm{H}_{1}$ lorsque son dosage dépasse une certaine valeur critique qui est pour notre cas de $25 \%$.

En effet, on considère que le mortier atteint sa compacité maximale lorsque le dosage critique en filler est atteint [11]; au-delà de ce dosage les frictions entre particules sont de plus en plus importantes.

Le tableau 7 montre que le ressuage diminue lorsque le dosage en fines est augmenté. Vraisemblablement parce que les particules fines s'hydratent plus rapidement et aussi parce que leur taux de sédimentation est plus faible. Donc les BAP fortement dosés en fines calcaires sont moins susceptibles de ressuer que les BAP maigres.

Tableau 7 : Influence du rapport $F / C$ sur la résistance à la ségrégation statique et le ressuage

\begin{tabular}{|l|c|c|c|}
\hline & $\mathrm{F} / \mathrm{C}=0,20$ & $\mathrm{~F} / \mathrm{C}=0,25$ & $\mathrm{~F} / \mathrm{C}=0,30$ \\
\hline Stabilité au tamis $\pi(\%)$ & 12,60 & 7,68 & 7,35 \\
\hline Ressuage $(\% 0)$ & $2,33$. & 1,15 & 1,05 \\
\hline
\end{tabular}

Les conclusions relatives aux compagnes d'essais sur l'essai de stabilité au tamis indiquent une bonne stabilité pour un pourcentage de laitance, $\pi$, inférieur à $15 \%$. Les résultats du Tableau 7 montrent que l'introduction de $20 \%$ à $30 \%$ de fines calcaires donne un BAP stable est homogène.

\section{3-4. Influence du volume de pâte}

Le volume de pâte est supposé jouer deux rôles dans un BAP. D'abord, il fluidifie le matériau en limitant les contacts entre les granulats, ensuite, il écarte suffisamment les 
gravillons pour éviter la formation d'amas contre les armatures, responsables de blocage de l'écoulement. Nous supposons qu'il existe un volume de pâte minimal permettant de remplir chacune de ces deux fonctions.

Parmi les bétons testés, un seul présente un étalement inférieur à $60 \mathrm{~cm}$ (Figure 7). On constate que l'augmentation du volume de pâte, contribue significativement à l'amélioration de la maniabilité. Cette amélioration est due principalement à la réduction de la teneur des gros granulats dont l'augmentation du volume et particulièrement les granulats concassés conduit à un frottement important [3]. C'est le cas du BAP au volume de pâte de $35 \%$. Sur le taux de remplissage, la Figure 8 montre une bonne corrélation entre le volume de pâte et le résultat de l'essai à la boîte en II $L$ II de nos bétons.

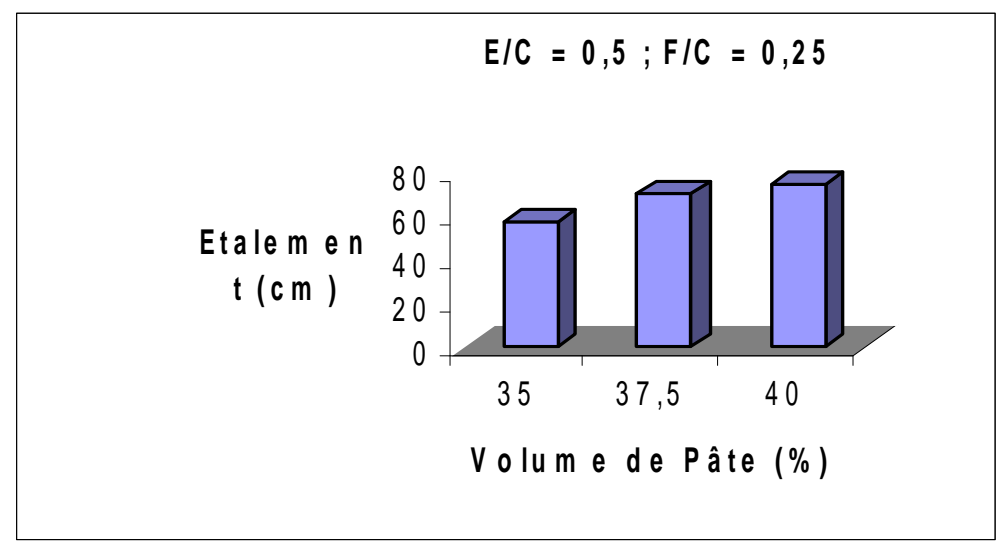

Figure 7 : Influence de la variation du volume de pâte sur le diamètre d'étalement

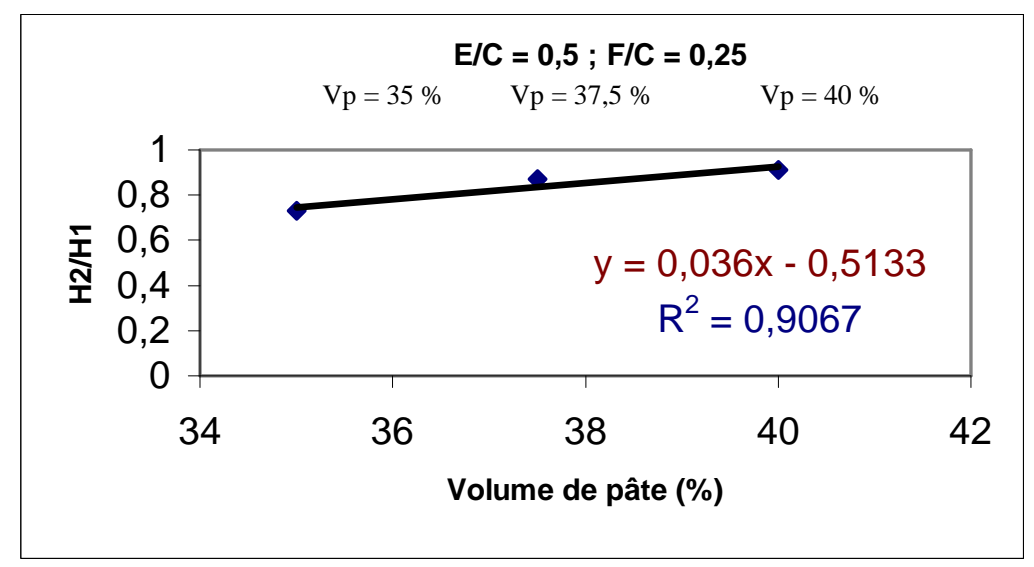

Figure 8 : Corrélation entre le taux de remplissage $\mathrm{H}_{2} / \mathrm{H}_{1}$ et le volume de pâte 
Le BAP au volume de pâte de $35 \%$ a présenté un blocage à la boite en II $L$ ॥ dont le taux de remplissage $H_{2} / H_{1}$ ne dépasse pas 0,80 . A cet effet on constate que pour une teneur en quantité de pâte de l'ordre de $35 \%$ avec un rapport $\mathrm{E} / \mathrm{C}$ de 0,5 et un rapport $\mathrm{F} / \mathrm{C}$ de 0,25 , ne nous permet pas d'obtenir un BAP répondant aux exigences de l'AFGC [6].

Le Tableau 8 montre les différents résultats obtenus des essais de stabilité au tamis et du ressuage.

Tableau 8 : Influence du volume de pâte sur la résistance à la ségrégation statique et le ressuage

\begin{tabular}{|l|c|c|c|}
\cline { 2 - 4 } \multicolumn{1}{l|}{ Caractéristique } & Vpâte $=35 \%$ & Vpâte $=37,5 \%$ & Vpâte = 40 \% \\
\hline Stabilité au tamis $\pi(\%)$ & 5,66 & 7,68 & 15,01 \\
\hline Ressuage (\%o) & 0,93 & 1,15 & 3,15 \\
\hline
\end{tabular}

Contrairement à l'essai d'étalement et la boîte en I $L$ II, le béton au volume de pâte de $40 \%$ se trouve à la limite du domaine autoplaçant puisque son pourcentage de laitance est légèrement supérieur à $15 \%$. Ce béton a également développé une stabilité critique vis-à-vis du ressuage dont le pourcentage a dépassé $3 \%$. Cette ségrégation est due principalement à l'augmentation du volume de pâte par rapport à celui des granulats.

\section{3-5. Influence du diamètre maximal des gravillons}

La taille maximale des granulats est aussi, un facteur non négligeable. En effet, plus la taille maximale des granulats augmente, plus le risque de blocage croit [12].

Afin de mettre en évidence l'influence de la taille maximale des granulats, deux proportions granulaires ont été considérées. II s'agit d'une combinaison volumique correspondant à la formulation de base (1) de $50 \%$ de sable (sable de mer+sable de carrière), $20 \%$ de la fraction $3 / 8$ et $30 \%$ de $8 / 15\left(\Phi_{\max }=15 \mathrm{~mm}\right)$, une autre qui présente une combinaison volumique de $50 \%$ de sable, $20 \%$ de la fraction $3 / 8,15 \%$ de $8 / 15$ et $15 \%$ de $15 / 20\left(\Phi_{\max }=20 \mathrm{~mm}\right)$. Le rapport $\mathrm{E} / \mathrm{C}$, le volume de pâte, le rapport $\mathrm{F} / \mathrm{C}$ et le dosage en superplastifiant sont gardés constants $(E / C=0,5 ;$ Vpâte $=37,5 \% ; F / C$ $=0,25 ; \mathrm{Sp}=1 \%$ ).

D'après le Tableau 9 , on trouve que la combinaison $(1)\left(\Phi_{\max }=15 \mathrm{~mm}\right)$ a favorisé les meilleures maniabilités et déformabilités par rapport à l'autre combinaison granulaire. On constate que le BAP au $\Phi_{\max }=20 \mathrm{~mm}$ est dans une limite autoplaçante basse; il est homogène et ne présente pas de risques de ségrégation statique et de ressuage, mais par contre il a un faible taux de remplissage et un étalement inférieur à $60 \mathrm{~cm}$. 
Tableau 9 : Influence du diamètre maximal des gravillons sur le comportement du BAP à l'état frais

\begin{tabular}{|l|c|c|}
\hline & $\Phi_{\max }=15 \mathrm{~mm}$ & $\Phi_{\max }=20 \mathrm{~mm}$ \\
\hline Etalement (cm) & 70,0 & 58,5 \\
\hline Taux de remplissage H2/HI & 0,87 & 0,73 \\
\hline Stabilité au tamis $\pi(\%)$ & 7,68 & 10,28 \\
\hline Ressuage (\%0) & 1,15 & 2,23 \\
\hline
\end{tabular}

On observe que la réduction de la taille des gros granulats, contribue significativement à l'amélioration de la maniabilité.

Cette amélioration est due principalement à la réduction de la teneur des gros granulats de la classe 15/20, car l'augmentation du diamètre des gravillons provoque un freinage surtout dans le cas des granulats concassés où les risques de frottement et de friction entre les particules sont importants à cause de leur morphologie. Les multiples frictions entre particules augmentent la résistance au déplacement du matériau ce qui provoque une augmentation de la viscosité du béton.

\section{3-6. Vérification de la ségrégation}

Un autre moyen de contrôler la ségrégation d'un BAP consiste à scier une ou (deux) éprouvette (s) de béton durci dans le sens transversal et observer la répartition des granulats dans les trois parties de l'éprouvette, le milieu, le bas et le haut (Photos 5, 6, 7 et 8)[4].
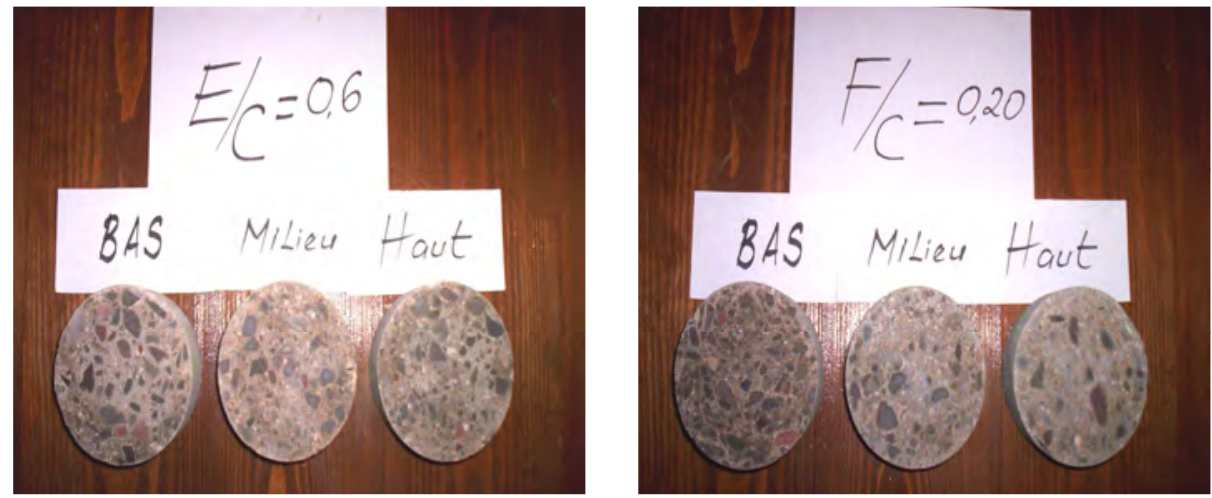

Photos 4 et 5 : Observation de la répartition des granulats dans les trois parties de I'éprouvette (BAP au rapport $E / C=0,6$ et $B A P$ au rapport $F / C=0,2)$ 

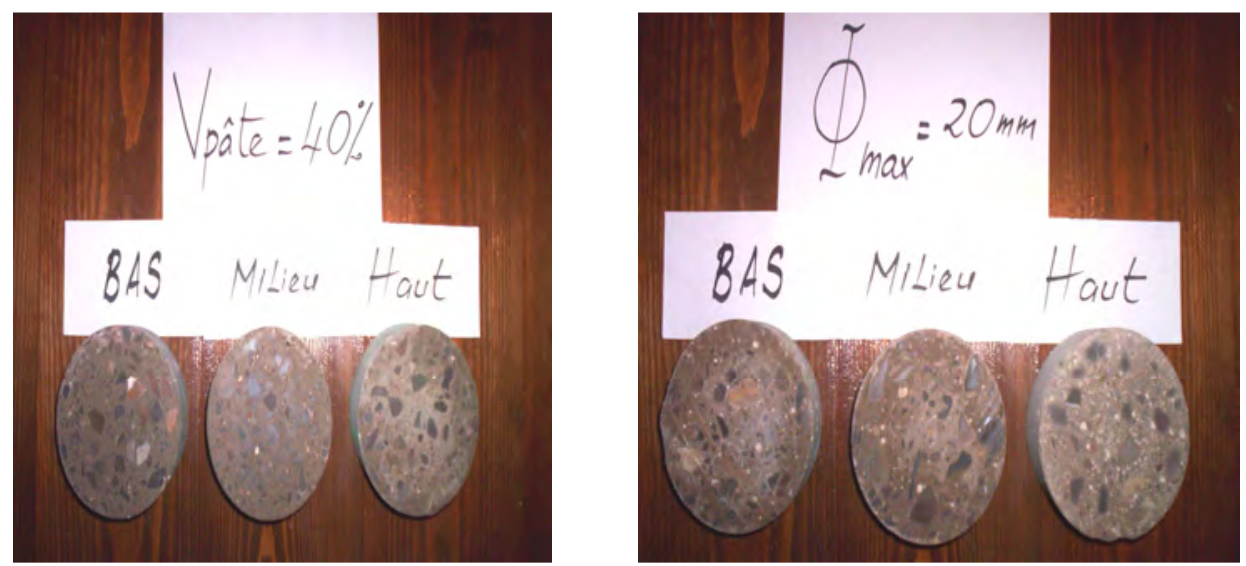

Photo 6 et 7 : Observation de la répartition des granulats dans les trois parties de l'éprouvette (BAP au volume de pâte Vpâte $=40 \%$ et BAP avec un diamètre maximal de granulats $\Phi_{\max }=20 \mathrm{~mm}$ )

Ces photographies illustrent bien le fait que les formulations de bétons présentées ici (BAP au rapport $\mathrm{E} / \mathrm{C}=0,6, \mathrm{BAP}$ au rapport $\mathrm{F} / \mathrm{C}=0,20, \mathrm{BAP}$ au volume de pâte Vpâte $=$ $40 \%$ et le BAP au diamètre maximale $\Phi_{\max }=20 \mathrm{~mm}$ ) sont effectivement sujettes à la ségrégation (statique et ou dynamique). Les granulats ne sont en effet régulièrement répartis sur toute la hauteur des échantillons sciés (coulés verticalement depuis le haut).

\section{Conclusion}

La présente recherche fait partie des premières expériences algériennes, au terme de celle-ci, nous pouvons noter qu'il est possible de fabriquer des BAP avec les matériaux locaux présentant les mêmes composants de base qu'un béton ordinaire et permettant ainsi d'obtenir les mêmes caractéristiques que celles connues à l'échelle internationale. Cependant les exigences que doit satisfaire un béton autoplaçant par rapport à un béton vibré sont sensiblement plus élevées en ce qui concerne sa formulation et sa caractérisation à l'état frais. La formulation du BAP requiert une étude précise notamment dans le choix des constituants, et l'optimisation des paramètres influant en particulier les rapports $E / C$ et $F / C$, le dosage en superplastifiant, le volume de pâte et le diamètre maximal des gravillons:

- Une teneur en superplastifiant de l'ordre de $0,5 \%$ avec $E / C=0,5, F / C=0,25$ et Vpâte $=37,5 \%$, la confection d'un BAP est quasiment impossible.

- Les rapports $E / C=0,4$ et $E / C=0,5(F / C=0,25$, Vpâte $=37,5 \%)$ ont influé 
positivement sur la stabilité et la résistance à la ségrégation du BAP.

- Le BAP de rapport $E / C=0,6$ (F/C et Vpâte constants) présente la particularité d'engendrer une mauvaise stabilité vis-à-vis de l'écoulement.

- L'ajout du filler calcaire contribue à diminuer la viscosité du BAP (augmentation du diamètre d'étalement).

- Lorsque le dosage en fines calcaires dépasse $25 \%$ (valeur critique), il provoque une augmentation de la viscosité se traduisant par une diminution du taux de remplissage $\mathrm{H}_{2} / \mathrm{H}_{1}$.

- Une teneur en quantité de pâte de l'ordre de $35 \%$ ( $E / C$ et $F / C$ constants) ne permet pas d'obtenir un BAP répondant aux exigences de l'AFGC.

- Le BAP au volume de pâte de 40 \% (E/C et $F / C$ constants) se trouve à la limite du domaine autoplaçant.

- Le BAP aux granulats de taille maximale $\Phi_{\max }=15 \mathrm{~mm}$ a favorisé les meilleurs maniabilités et déformabilités.

- Le BAP au $\Phi_{\max }=20 \mathrm{~mm}$ se trouve dans une limite autoplaçante basse.

Pour appuyer notre recherche, une deuxième partie sera entamée. II s'agit de l'étude de I'influence des paramètres de composition sur le comportement du BAP vis-à-vis du retrait. Divers essais relatifs aux domaines mécaniques et celui de la microstructure seront réalisés.

\section{Références}

[1] - H. Okamura, M. Ouchi "Self-compacting concrete. Development present use and future", First International RILEM symposium on self-compacting concrete. Rilem Publication s.a.r.l, (1999) 3-14

[2] - Noor MA., T. Uomoto "Three-dimensional discrete element simulation of rheology tests of self-compacting concrete", First International RILEM symposium on selfcompacting concrete, RILEM Publications s.a.r.I, (1999) 35-46

[3] - A. Neville ॥ Propriétés des bétons ॥, Centre de Recherche International du Béton, Editions Eyrolles (2000).

[4] - S. Assie " Durabilité des bétons autoplaçants ॥, Thèse de l'INSA de Toulouse, $\mathrm{N}^{\circ} 747$ (2004).

[5] - T. Sedran, F. de Larrard ॥ Manuel d'utilisation de RENE-LCPC, Logiciel d'optimisation granulaire II, version 6.1 d, Laboratoire Central des Ponts et Chaussées (LCPC) (2000).

[6] - Association Française de Génie Civil (AFGC) "Les bétons autoplaçants \#, Recommandations provisoires AFGC, (2000). 
[7] - E. Proust ॥ Retrait et fluage des bétons autoplaçants : vers une compréhension des comportements différés II, thèse de doctorat de I'Institut National des Sciences Appliquées, Toulouse, $\mathrm{N}^{\circ} 137,(2002)$.

[8] - G. Bonneau ॥ Etude des effets physico-chimiques des superplastifaints en vue d'optimiser le comportement rhéologique des bétons à Ultra-Hautes Performances II, thèse de doctorat en cotutelle, Ecole Nationale Supérieure de Cachan et Université de Sherbrooke, (1997).

[9] - S. G. Oh, T. Nogushi and F. Tomosawa "Toward mix design for rheologie of selfcompacting concrete", In Proceedings of the First International RILEM Symposium of Self-Compacting Concrete, RILEM, 1999.

[10] - S. Utsi, M. Emborg, J. Carsward "Relation between workability and theological parameters", Therd International RILEM symposium on self-compacting concrete, RILEM Publication s.a.r.l, (2003) 311-322

[11] - A. Yahia, M. Tanimura, Y. Shimoyama "Rheological properties of highly flowable mortar containing limestone filler-effect of powder content and $W / C$ ratio", Cement and Concrete Research, CCR journa/35 (3) (2005) 532-539

[12] - M. Yurugi, N. Sakata, M. Iwai and G. Sakai "Mix Proportion for Highly Workable Concrete", Conference Concrete, Dundee, 7-9 September, (2000) 325-332 